Editor's Note: These short reviews of a recent paper in the Journal, written exclusively by graduate students or postdoctoral fellows, are intended to mimic the journal clubs that exist in your own departments or institutions. For more information on the format and purpose of the Journal Club, please see http://www.jneurosci.org/misc/ifa_features.shtml.

\title{
Labeled-Line Coding and Summation Coding of Numerosities in Prefrontal and Parietal Cortex
}

\author{
Filip Van Opstal \\ Department of Experimental Psychology, Ghent University, B-9000 Ghent, Belgium \\ Review of Nieder and Merten (http://www.jneurosci.org/cgi/content/full/27/22/5986)
}

Computational models in humans suggest two different types of coding numerical quantity (Dehaene and Changeux, 1993). Summation coding reflects the idea that numerical quantity (or numerosity) is encoded by a monotonically increasing response function: the higher the numerosity, the more neuronal activation. In labeled-line coding (or place coding) a specific neuron responds most strongly to one specific numerosity [Nieder and Merten (2007), their Fig. 1 (http://www. jneurosci.org/cgi/content/full/27/22/5986/ F1)]. The evidence for these different types of encoding is relatively sparse. On a behavioral level, it has only recently been shown that both types of encoding coexist, and that the coding system depends on the stimulus material used (dot patterns or Arabic numerals) (Roggeman et al., 2007). On a neuronal level, several singlecell studies have revealed labeled-line coding neurons (Nieder and Miller, 2004): the firing rate of prefrontal and parietal neurons in rhesus monkeys responded maximally to specific numerosities. Although these neurons behave exactly as described by labeled-line coding, extreme numerosities (1 and 5) might act as low-

Received June 7, 2007; revised July 24, 2007; accepted July 24, 2007.

F.V.0. was supported by Fund of Scientific Research, Flanders Grant G.0188.04.

Correspondence should be addressed to Filip Van 0pstal, Department of Experimental Psychology, Ghent University, Henri Dunantlaan 2, B-9000, Ghent, Belgium. E-mail: filip.vanopsta@ugent.be.

DOI:10.1523/JNEUROSCI.2592-07.2007

Copyright $\odot$ 2007 Society for Neuroscience $\quad$ 0270-6474/07/279535-02\$15.00/0 and high-pass filters (Roggeman et al., 2007). Furthermore, because all neurons had identical short firing-rate latencies for all numerosities tested (ranging from 1 to $5)$, the extraction of numerosity must have been parallel across the entire display (Nieder and Miller, 2004). This raised the possibility that the number selectivity of the neurons was a feature of the subitizing mechanism and that numerosity might be calculated in the parietal cortex and then forwarded to the prefrontal cortex (PFC) to be held in memory (Feigenson et al., 2004).

In a new study published in The Journal of Neuroscience, Nieder and Merten (2007) show that labeled-line coding is also present for larger numerosities. Two rhesus monkeys were trained to perform the delayed match-to-numerosity task. In this task, monkeys are trained to judge whether two successive displays contain the same number of randomly placed dots. The numerosity of dots on a display ranged from 1 to 30 . Because the presented dot patterns are controlled for area and density, the monkeys must extract the numerosity of the set of dots to complete the task. The results mirror those obtained with small numerosities (Nieder and Miller, 2004). The behavioral data showed both an effect of distance (more errors are made when the numerosities on the successive displays are numerically close) and size (more errors are made with larger numerosities), following the same psychophysical laws that sensory judgments follow [Nieder and Merten (2007), their Fig. 3 (http://www. jneurosci.org/cgi/content/full/27/22/5986/ F3)]. The distributions of the responses were symmetric when plotted on a logarithmic scale, suggesting a nonlinear coding scheme. Importantly, the neuronal data showed that the PFC contained neurons with preferred numerosities for the complete range between 1 and 30 [Nieder and Merten (2007), their Fig. 5 (http://www. jneurosci.org/cgi/content/full/27/22/5986/ F5)].

These results clearly demonstrate the presence of an analog magnitude system with labeled-line coding in a numbermatching task. The finding that numberselective neurons can code for numerosities up to 30 shows that the analog magnitude system can represent a large number of different numerosities. Furthermore, the importance of labeled-line coding neurons for task performance was made explicit by showing that the neural activity on incorrect trials for the preferred neuron was significantly reduced during the sample and delay epochs.

However, as suggested by Nieder and Merten (2007), these results could be specific to the task used. In a numbermatching task, monkeys have to decide whether the sample and the test numerosities are the same or different. Because a same/different task is not linearly separable, labeled-line encoding might be a more efficient way of coding for this specific task (Verguts, 2007), but that does not exclude the possibility of summation 
coding. Indeed, computational models of number processing have suggested that summation coding precedes labeled-line coding (Dehaene and Changeux, 1993). Summation coding could then be seen as a first, more natural way of extracting numerosity. Evidence for this assumption comes from Roitman et al. (2007). In their study, an implicit numerical discrimination task with dot patterns ranging from 2 to 32 was used. Although numerosity processing was irrelevant to complete the task, their results showed that monkeys performed an implicit numerosity comparison. More importantly, however, the neural data revealed that, during the presentation of the numerosity, firing rates from neurons in lateral intraparietal sulcus monotonically increased or decreased when numerosity increased and thus clearly exhibited summation coding. Because there was no numerical task, a labeled-line code might not have been developed during training.
In sum, the new results from Nieder and Merten (2007) together with the results of Roitman et al. (2007), as well as a previous observation that numberselective neurons in the PFC could be related to memory storage (Nieder and Miller, 2004), suggest that primary numerosity extraction occurs in the parietal cortex using summation coding. Depending on the task, the numerosity information is then encoded in a labeled-line code and forwarded to the PFC to be stored in memory. To further investigate the properties of this parietofrontal network, it might be interesting to apply the numbermatching task and record from cells in parietal cortex. Furthermore, to study the relationship between number-selective neurons and the nonlinearity of the number-matching task, it could also be interesting to investigate neurons in the PFC during the presentation of numerosities in the implicit number discrimination task used by Roitman et al. (2007).

\section{References}

Dehaene S, Changeux JP (1993) Development of elementary numerical abilities: a neuronal model. J Cogn Neurosci 5:390-407.

Feigenson L, Dehaene S, Spelke E (2004) Core systems of number. Trends Cogn Sci 8:307-314

Nieder A, Merten K (2007) A labeled-line code for small and large numerosities in the monkey prefrontal cortex. J Neurosci 27:5986-5993.

Nieder A, Miller EK (2004) A parieto-frontal network for visual numerical information in the monkey. Proc Natl Acad Sci USA 101:7457-7462.

Roggeman C, Verguts T, Fias W (2007) Priming reveals differential coding of symbolic and non-symbolic quantities. Cognition, in press.

Roitman JD, Brannon EM, Platt ML (2007) Monotonic coding of numerosity in macaque lateral intraparietal area. PLoS Biol 5:e208.

Verguts T (2007) How to compare two quantities? A computational model of flutter discrimination. J Cogn Neurosci 19:409-419. 